\title{
A NEW FLAG TRANSITIVE AFFine PLANE OF ORDER 27
}

\author{
M. L. NARAYANA RAO AND K. KUPPUSWAMY RAO
}

\begin{abstract}
A flag transitive affine plane of order 27 is constructed. The stabilizer of the origin of this plane contains a cyclic subgroup which is transitive on the lines through the origin. It is also shown that this plane is not isomorphic to the flag transitive plane constructed by Hering.
\end{abstract}

1. Let $\pi$ be a finite affine plane of order $n$. A collineation group $G$ of $\pi$ is defined to be flag transitive on $\pi$ if $G$ is transitive on incident point-line pairs or flags of $\pi$. A. Wagner [13] has shown that $\pi$ is a translation plane so that $n=p^{r}$ for some prime $p$ and for some integer $r>0$. D. A. Foulser [4], [5] has determined all flag transitive groups of finite affine planes. While determining the flag transitive groups, Foulser remarks that the existence of non-Desarguesian flag transitive affine planes is still an open problem. However, Foulser constructs two flag transitive planes of order 25 [4] and shows that his two planes and the near field plane of order 9 have flag transitive collineation groups. C. Hering [7] has constructed a plane of order 27 which has a flag transitive collineation group. Recently, one of the authors [10], [11] has constructed a flag transitive plane of order 49 and a class of flag transitive planes of order $q^{2}$ where $q$ is power of a prime $p>3$. The aim of this paper is to construct a non-Desarguesian flag transitive plane of order 27 and establish that this is different from the plane constructed by Hering [7].

2. Let $F$ be the set of all ordered triples $(a, b, c)$ over $G F(3)$. Let $C$ be a set of $3 \times 3$ matrices over $G F(3)$ satisfying:

(2.1) (i) $C$ contains

$$
\left(\begin{array}{lll}
0 & 0 & 0 \\
0 & 0 & 0 \\
0 & 0 & 0
\end{array}\right) \text { and }\left(\begin{array}{ccc}
1 & 0 & 0 \\
0 & 1 & 0 \\
0 & 0 & 1
\end{array}\right) \text {, }
$$

(ii) $C$ contains 27 matrices,

(iii) If $M, N \in C$ and $M \neq N$, then $|M-N| \neq 0$, where $|X|$ denotes determinant of matrix $X$.

The conditions $(2.1)$ imply that corresponding to each ordered triple $(a, b, c)$ in $F$, there is exactly one matrix of the form

Received by the editors October 17, 1974.

AMS (MOS) subject classifications (1970). Primary 50D30, 05B25; Secondary 15B25.

Key words and phrases. Projective planes, affine planes, flag transitivity, collineations. 


$$
\left(\begin{array}{lll}
a & b & c \\
p & q & r \\
x & y & z
\end{array}\right)
$$

which will be denoted by $M(a, b, c)$. We now define addition "+" and multiplication "." on $F$ as follows.

$$
\begin{aligned}
(a, b, c)+(d, e, f) & =(a+d, b+e, c+f), \\
(a, b, c) \cdot(d, e, f) & =(d, e, f) M(a, b, c)
\end{aligned}
$$

where $M(a, b, c)$ is the matrix in $C$ corresponding to $(a, b, c)$.

THEOREM 2.1. The set $F$ with operations + and $\cdot$ defined by (2.2) is a left Veblen-Wedderburn system.

Proof. See $[3, \S 5]$ or $[2, \S 5]$.

3. We now construct a set $C$ of matrices over $G F(3)$ and show that $C$ satisfies conditions (2.1).

Let $T=\left(\begin{array}{ll}0 & P \\ Q & R\end{array}\right)$ be a $6 \times 6$ matrix over $G F(3)$ where 0 is $3 \times 3$ zero matrix and $P, Q, R$ are $3 \times 3$ nonsingular matrices given by

$$
P=\left(\begin{array}{lll}
1 & 1 & 1 \\
0 & 2 & 1 \\
2 & 2 & 0
\end{array}\right), \quad Q=\left(\begin{array}{lll}
0 & 2 & 1 \\
0 & 0 & 1 \\
1 & 2 & 0
\end{array}\right), \quad R=\left(\begin{array}{lll}
1 & 2 & 1 \\
0 & 0 & 2 \\
2 & 0 & 2
\end{array}\right)
$$

Let

$$
M_{0}=\left(\begin{array}{lll}
0 & 0 & 0 \\
0 & 0 & 0 \\
0 & 0 & 0
\end{array}\right)
$$

Matrices $M_{i}, 2 \leqslant i \leqslant 27$, are inductively defined as

$$
\begin{array}{r}
M_{2}=Q^{-1} R=\left(\begin{array}{lll}
1 & 1 & 0 \\
2 & 1 & 1 \\
0 & 0 & 2
\end{array}\right), \quad M_{k+1}=Q^{-1} M_{k}^{-1} P+M_{2}, \\
2 \leqslant k \leqslant 26 .
\end{array}
$$

An inspection of Table 3.1 shows that for each $k, 3 \leqslant k<26, M_{k-1}$ is nonsingular and, therefore, $M_{k}$ is defined for $3 \leqslant k \leqslant 27$.

Lemma 3.1. The set $C=\left\{M_{i} \mid i=0,2 \leqslant i \leqslant 27\right\}$ satisfies the conditions (2.1) and, hence, the corresponding $F(+, \cdot)$ is a left Veblen-Wedderburn system.

Proof. An inspection of Table 3.1 shows that $C$ contains the zero and the unit matrices, the nonzero matrices of $C$ are nonsingular and $C$ consists of 27 matrices in all. Further, $Q^{-1} M_{27}^{-1} P+Q^{-1} R=M_{0}$. From (3.1) we obtain

$$
\left|M_{k+1}-M_{2}\right|=\left|Q^{-1}\right|\left|M_{k}^{-1}\right||R| \neq 0
$$

since $Q, R$ and $M_{k}$ are nonsingular for $2 \leqslant k \leqslant 26$. Since $M_{0}$ is the $3 \times 3$ zero matrix and $M_{k}$ is nonsingular for $2 \leqslant k \leqslant 27$, 
TABLE 3.1

\begin{tabular}{|c|c|c|c|c|c|}
\hline$i$ & $M_{i}$ & $i$ & $M_{i}$ & $i$ & $M_{i}$ \\
\hline 0 & $\begin{array}{lll}0 & 0 & 0 \\
0 & 0 & 0 \\
0 & 0 & 0\end{array}$ & 10 & $\begin{array}{lll}2 & 0 & 0 \\
1 & 0 & 2 \\
1 & 2 & 1\end{array}$ & 19 & $\begin{array}{lll}0 & 1 & 1 \\
1 & 1 & 0 \\
1 & 2 & 2\end{array}$ \\
\hline 2 & $\begin{array}{lll}1 & 1 & 0 \\
2 & 1 & 1 \\
0 & 0 & 2\end{array}$ & 11 & $\begin{array}{lll}0 & 2 & 2 \\
2 & 2 & 2 \\
2 & 0 & 2\end{array}$ & 20 & $\begin{array}{lll}2 & 2 & 0 \\
2 & 0 & 0 \\
0 & 2 & 2\end{array}$ \\
\hline 3 & $\begin{array}{lll}1 & 0 & 1 \\
1 & 2 & 2 \\
0 & 1 & 0\end{array}$ & 12 & $\begin{array}{lll}2 & 1 & 2 \\
0 & 2 & 0 \\
2 & 0 & 1\end{array}$ & 21 & $\begin{array}{lll}2 & 1 & 1 \\
1 & 1 & 2 \\
2 & 1 & 2\end{array}$ \\
\hline 4 & $\begin{array}{lll}1 & 2 & 2 \\
1 & 2 & 0 \\
2 & 2 & 2\end{array}$ & 13 & $\begin{array}{lll}0 & 1 & 2 \\
2 & 0 & 1 \\
0 & 1 & 1\end{array}$ & 22 & $\begin{array}{lll}2 & 2 & 1 \\
2 & 1 & 0 \\
2 & 0 & 0\end{array}$ \\
\hline 5 & $\begin{array}{lll}0 & 0 & 2 \\
2 & 2 & 0 \\
1 & 2 & 0\end{array}$ & 14 & $\begin{array}{lll}1 & 0 & 0 \\
0 & 1 & 0 \\
0 & 0 & 1\end{array}$ & 23 & $\begin{array}{lll}1 & 2 & 0 \\
2 & 0 & 2 \\
1 & 0 & 0\end{array}$ \\
\hline 6 & $\begin{array}{lll}1 & 1 & 2 \\
0 & 2 & 1 \\
2 & 1 & 0\end{array}$ & 15 & $\begin{array}{lll}2 & 1 & 0 \\
1 & 2 & 1 \\
0 & 2 & 0\end{array}$ & 24 & $\begin{array}{lll}1 & 2 & 1 \\
1 & 0 & 0 \\
1 & 1 & 1\end{array}$ \\
\hline 7 & $\begin{array}{lll}0 & 1 & 0 \\
1 & 0 & 1 \\
0 & 2 & 1\end{array}$ & 16 & $\begin{array}{lll}0 & 2 & 0 \\
0 & 2 & 2 \\
1 & 1 & 2\end{array}$ & 25 & $\begin{array}{lll}0 & 2 & 1 \\
1 & 1 & 1 \\
2 & 2 & 0\end{array}$ \\
\hline 8 & $\begin{array}{lll}2 & 0 & 2 \\
0 & 0 & 2 \\
1 & 1 & 0\end{array}$ & 17 & $\begin{array}{lll}0 & 0 & 1 \\
0 & 1 & 2 \\
2 & 2 & 1\end{array}$ & 26 & $\begin{array}{lll}1 & 1 & 1 \\
0 & 0 & 1 \\
1 & 0 & 2\end{array}$ \\
\hline 9 & $\begin{array}{lll}2 & 2 & 2 \\
0 & 1 & 1 \\
0 & 1 & 2\end{array}$ & 18 & $\begin{array}{lll}2 & 0 & 1 \\
2 & 2 & 1 \\
1 & 0 & 1\end{array}$ & 27 & $\begin{array}{lll}1 & 0 & 2 \\
2 & 1 & 2 \\
2 & 1 & 1\end{array}$ \\
\hline \multicolumn{6}{|c|}{$\left|M_{k}-M_{0}\right| \neq 0$} \\
\hline
\end{tabular}

Let $1 \leqslant k \leqslant 26,1 \leqslant j \leqslant 26, k \neq j$. There is no loss of generality if we assume $k>j$. From (3.1) we obtain

$$
M_{k+1}-M_{j+1}=Q^{-1} M_{k}^{-1}\left(M_{j}-M_{k}\right) M_{j}^{-1} R
$$

which implies that

$$
\left|M_{k+1}-M_{j+1}\right|=\mu(j, k)\left|M_{j}-M_{k}\right|
$$

where $\mu(j, k)=\left|Q^{-1}\right|\left|M_{k}^{-1}\right|\left|M_{j}^{-1}\right||R| \in G F(3)$ and $\mu(j, k) \neq 0$. Applying (3.4) repeatedly we obtain

$$
\left|M_{k+1}-M_{j+1}\right|=\mu\left|M_{k-j+2}-M_{2}\right|
$$

where $\mu$ is a nonzero element of $G F(3)$ which depends upon $k$ and $j$. In view 
of (3.2) we get

$$
\left|M_{k+1}-M_{j+1}\right| \neq 0 \text {. }
$$

Now the lemma follows from (3.2), (3.3), (3.6) and Theorem 2.1.

4. Let $\Pi_{N}$ be the projective plane coordinatized by $F(+, \cdot)[4$, p. 353], [6, p. 45], [10, p. 31]. That is $\Pi_{N}$ has points $(c),(a, d),(\infty)$ and lines $[k],[m, b]$, $[\infty]=L_{\infty}$ for $a, b, c, d, k, m \in F$ and $\infty \notin F$. Incidence in $\Pi_{N}$ is defined by $(x, y) \in[m, b]$ if $y=m \cdot x+b$ and $(x, y) \in[k]$ if $x=k$. The plane $\Pi_{N}$ may also be considered as a six dimensional right vector space $V(6,3)$ with three dimensional subspaces of $V(6,3)$ as lines and vectors of $V(6,3)$ as points. The line corresponding to the equation $y=m \cdot x, m \in F$, is given by the subspace

$$
V(m)=\{(a, b, c, d, e, f) \mid(a, b, c) \in F,(d, e, f)=(a, b, c) M(m)\}
$$

where $M(m)$ is the unique matrix in $C$ corresponding to $m \in F$. The line $x=0$ corresponds to the subspace

$$
V(\infty)=\{(0,0,0, a, b, c) \mid(a, b, c) \in F\} .
$$

The lines $y=m \cdot x+b$ correspond to appropriate translates of $V(m)$ for $m \in F$ or $m=\infty$. The group $G_{0}$ of all collineations fixing $(0,0,0)$ of $\Pi_{N}$ consists of all nonsingular linear transformations of $V(6,3)$ which permute the subspaces $V(m)$ for $m \in F$ or $m=\infty$ among themselves [1, Satz 19], [12, p. 208].

We now show that $T$ given in $\S 3$ is a collineation of $\Pi_{N}$ fixing the point corresponding to zero vector in $V(6,3)$. For convenience let the subspace $V(m)$ be denoted by $V_{k}$, where $M_{k}$ is the unique matrix in $C$ corresponding to $m \in F$, and $V(\infty)$ be denoted by $V_{1}$. It is clear that $T$ is nonsingular. Since $P$ is nonsingular it is easy to see that $V_{0} T=V_{1}$. Now

$$
\begin{aligned}
V_{1} T=\{(0,0,0, a, b, c) \mid(a, b, c) & \in G F(3)\} T \\
=\{(x, y, z, p, q, r) \mid(x, y, z) & =(a, b, c) Q \\
\text { and }(p, q, r) & =(a, b, c) R,(a, b, c) \in G F(3)\} \\
=\{(x, y, z, p, q, r) \mid(p, q, r) & =(x, y, z) Q^{-1} R \\
& \left.=(x, y, z) M_{2},(x, y, z) \in G F(3)\right\} \\
= & V_{2} .
\end{aligned}
$$

Similarly, in view of relations

$$
M_{i+1}=Q^{-1} M_{i}^{-1} P+Q^{-1} R \quad \text { and } \quad M_{0}=Q^{-1} M_{27}^{-1} P+Q^{-1} R,
$$

we obtain that $V_{i} T=V_{k}$ where $k \equiv i+1(\bmod 28)$ for $2 \leqslant i \leqslant 27$. Applying $T$ repeatedly we obtain that

$$
V_{i} T^{j}=V_{k} \quad \text { where } k \equiv i+j \quad(\bmod 28) .
$$

Thus $\langle T\rangle$ is a collineation group of $\Pi_{N}$ which permutes the lines through the 
origin transitively. The action of $T$ restricted to the lines through the origin of $\Pi_{N}$ may be given by $T:(0,1,2,3, \ldots, 27)$ where $x$ stands for the line $V_{x}$. Let $\pi_{N}$ be the affine plane obtained from $\Pi_{N}$ by deleting the line containing the ideal points.

THEOREM 4.1. The plane $\pi_{N}$ is a non-Desarguesian flag transitive affine plane.

Proof. An examination of Table 3.1 reveals that the set $C=\left\{M_{i} \mid i=0,2\right.$ $\leqslant i \leqslant 27\}$ of matrices does not form a Galois field under matrix addition and multiplication implying $\Pi_{N}$ is non-Desarguesian [3, p. 230]. Thus $\pi_{N}$ is nonDesarguesian. The rest of the theorem follows from the action of $T$ restricted to the lines of $\pi_{N}$ through the origin.

Lemma 4.2. Any collineation of $\pi_{N}$ fixing $V_{0}, V_{1}, V_{14}, V_{15}$ and permuting the other lines through the origin among themselves fixes $V_{7}$ also.

Proof. Let $S=\left(\begin{array}{c}U V \\ W X\end{array}\right)$, where $U, V, W, X$ are $3 \times 3$ matrices over $G F(3)$, be a matrix inducing a collineation of $\pi_{N}$ fixing $V_{0}, V_{1}, V_{14}, V_{15}$ and permuting the other lines of $\pi_{N}$ through the origin. Since $S$ fixes $V_{0}$ and $V_{1}, S$ reduces to the form $\left(\begin{array}{ll}U & 0 \\ 0 & X\end{array}\right)$ where 0 is $3 \times 3$ zero matrix, and $U$ and $X$ are $3 \times 3$ nonsingular matrices [9, Lemma 1.2]. Since $S$ permutes the lines $V_{i}, 2 \leqslant i \leqslant 27$, among themselves, $U$ and $X$ must be such that for each $M_{i} \in C$ there must be a $M_{j} \in C$ satisfying $U M_{i}=M_{j} X, i \neq 0,1, J \neq 0,1$. Obviously, $S$ fixes $V_{i}$ if and only if $U M_{i}=M_{i} X$. Since $S$ fixes $M_{14}$ and $M_{14}=I$, the $3 \times 3$ unit matrix, we get that $U=X$. But $S$ fixes $V_{15}$ also so that $U M_{15} U^{-1}=M_{15}$. An examination of Table 3.1 shows that

$$
M_{7}=M_{14}+M_{15}=I+M_{15} .
$$

Then

$$
U M_{7} U^{-1}=U I U^{-1}+U M_{15} U^{-1}=I+M_{15}=M_{7} .
$$

Thus $S$ fixes $V_{7}$ also. Hence, the lemma.

5. We give a brief description of the construction of Hering's affine plane $\pi_{H}$ [7] and deduce some of its collineations in order to compare it with the plane $\pi_{N}$. Let 0 be $3 \times 3$ zero matrix. Let

$$
\begin{aligned}
& s=\left(\begin{array}{cc}
A_{1} & 0 \\
0 & A_{2}
\end{array}\right) \quad \text { where } A_{1}=\left(\begin{array}{lll}
0 & 2 & 0 \\
2 & 0 & 2 \\
1 & 0 & 0
\end{array}\right), \quad A_{2}=\left(\begin{array}{lll}
2 & 0 & 1 \\
2 & 0 & 0 \\
0 & 2 & 0
\end{array}\right), \\
& r=\left(\begin{array}{ll}
B_{1} & B_{2} \\
B_{3} & B_{4}
\end{array}\right) \quad \text { where } B_{1}=\left(\begin{array}{lll}
2 & 0 & 0 \\
2 & 1 & 0 \\
1 & 2 & 2
\end{array}\right), \quad B_{2}=\left(\begin{array}{lll}
2 & 0 & 0 \\
2 & 1 & 0 \\
1 & 2 & 2
\end{array}\right), \\
& B_{3}=\left(\begin{array}{lll}
2 & 0 & 0 \\
2 & 1 & 0 \\
2 & 2 & 2
\end{array}\right), \quad B_{4}=\left(\begin{array}{lll}
1 & 0 & 0 \\
1 & 2 & 0 \\
2 & 1 & 1
\end{array}\right) \\
& h=\left(\begin{array}{cc}
0 & C_{1} \\
C_{2} & 0
\end{array}\right) \quad \text { where } C_{1}=\left(\begin{array}{lll}
2 & 0 & 0 \\
2 & 2 & 0 \\
0 & 1 & 2
\end{array}\right), \quad C_{2}=\left(\begin{array}{lll}
1 & 0 & 0 \\
1 & 1 & 0 \\
2 & 2 & 1
\end{array}\right) \text {. }
\end{aligned}
$$


Let $L_{26}$ and $L_{27}$ be three dimensional subspaces of $V(6,3)$ defined by basis vectors as

$$
\begin{aligned}
& L_{26}=\langle(1,0,0,0,0,0),(0,1,0,0,0,0),(0,0,1,0,0,0)\rangle, \\
& L_{27}=\langle(0,0,0,1,0,0),(0,0,0,0,1,0),(0,0,0,0,0,1)\rangle .
\end{aligned}
$$

Let

$$
L_{i}=L_{26} r s^{i}, \quad 0 \leqslant i \leqslant 12, \quad L_{13+i}=L_{27} r s^{i}, \quad 0 \leqslant i \leqslant 12 .
$$

The incidence structure $\pi_{H}$ with $L_{i}(0 \leqslant i \leqslant 27)$ and their cosets in the additive group of $V(6,3)$ as lines and vectors of $V(6,3)$ as points with inclusion as incidence relation is the flag transitive plane of order 27 constructed by Hering [7].

We will now show that $s, h$ and $r$ induce collineations on $\pi_{H}$. It is easy to verify that

$L_{26} s=L_{26}$ and $L_{27} s=L_{27}$ and

$L_{i} s^{j}=L_{k}$ where $k \equiv i+j(\bmod 13), 0 \leqslant i \leqslant 12,0 \leqslant j \leqslant 12$, and

$L_{13+i} s^{j}=L_{13+k}$ where $k \equiv i+j(\bmod 13)$.

Thus $s$ induces a collineation on $\pi_{H}$ the action of which restricted to lines through the origin is given by $s:(26)(27)(0,1,2, \ldots, 12)(13,14, \ldots, 25)$. Here $(x)$ stands for line $L_{x}$.

In order to consider actions of $h$ and $r$ on $\pi_{H}$ we need the following relations between generators $r, h$ and $s$ of the group $G$ considered by Hering [7].

$$
s^{13}=I, \quad h^{6}=r^{2}=2 I, \quad r^{-1} h r=h^{-1},
$$

where $I$ is the $6 \times 6$ unit matrix.

$$
h^{-1} s h=s^{4}, \quad r^{-1} s r=s^{-1} r s^{-1} .
$$

For $1<i \leqslant 12, r^{-1} s^{i} r$ can be computed according to the relations given by Hering [7, p. 205].

It can be checked that

$$
L_{26} h=L_{27}, \quad L_{27} h=L_{26} \quad \text { and } \quad L_{0} h=L_{26} r h=L_{26} h^{-1} r=L_{27} r=L_{13} .
$$

Similarly, $L_{13} h=L_{0}$.

Let $1<i \leqslant 12$. Then

$$
\begin{aligned}
L_{i} h & =L_{26} r s^{i} h=L_{26} r h h^{-1} s^{i} h=L_{26} r h s^{4 i} \\
& =L_{26} h^{-1} r s^{4 i}=L_{27} r s^{4 i}=L_{13} s^{4 i}=L_{13+(4 i \bmod 13)}
\end{aligned}
$$

Similarly, $L_{13+i} h=L_{4 i(\bmod 13)}$.

Thus it follows that $h$ induces a collineation and its action restricted to the lines of $\pi_{H}$ through origin is

$$
h:(26,27),(0,13),(1,17,3,25,9,23),(2,21,6,24,5,20),
$$

$$
(4,16,12,22,10,14),(7,15,8,19,11,18) \text {. }
$$

The action of $h^{2}$ restricted to the lines $\pi_{H}$ is given by 


$$
\begin{gathered}
h^{2}:(26)(27)(0)(13)(1,3,9)(2,6,5)(4,12,10)(7,8,11)(14,16,22) \\
(15,19,18)(17,25,23)(20,21,24) .
\end{gathered}
$$

From the definition of $L_{0}$ and $L_{13}$ we get $L_{26} r=L_{0}$ and $L_{27} r=L_{13}$. Since $r^{2}=2 I, L_{0} r=L_{26} r^{2}=L_{26}$. Similarly $L_{13} r=L_{27}$. From $r^{-1} s r=s^{12} r s^{12}$ it follows that

$$
L_{1} r=L_{26} r s r=L_{26} r^{2} r^{-1} s r=L_{26} s^{12} r s^{12}=L_{12} .
$$

Here we have used the facts that $L_{26} r^{2}=L_{26}$ and $L_{26} s^{i}=L_{26}$ for any $i$. Similarly, using conjugates of $s^{i}$ by $r$ and noting that $h$ interchanges $L_{26}$ and $L_{27}$, we may conclude that $r$ induces a collineation and its action restricted to the lines $L_{i}$ is

$$
\begin{gathered}
r:(0,26)(13,27)(1,12)(2,19)(3,4)(5,18)(6,15)(7,24)(8,21)(9,10) \\
(11,20)(14,25)(16,17)(22,23) .
\end{gathered}
$$

Theorem 5.1. Any collineation of $\pi_{H}$ that fixes $L_{26}$ also fixes $L_{27}$.

Proof. Let $\beta$ be any collineation of $\pi_{H}$ fixing $L_{26}$ and moving $L_{27}$ to $L_{x}$, $0 \leqslant x \leqslant 12$. Since $s$ fixes $L_{26}$ and is transitive on the lines $L_{i}, 0 \leqslant i \leqslant 12$, we may as well take $\beta$ such that $\beta$ fixes $L_{26}$ and maps $L_{27}$ onto $L_{0}$. Then the action of

$$
\beta^{-1} s \beta=(26)(0)\left(x_{0}, x_{1}, \ldots, x_{12}\right)\left(y_{13}, \ldots, y_{25}\right),
$$

where $L_{x_{i}}=L_{i} \beta ; L_{y_{13+i}}=L_{13+i} \beta$. Now two cases need to be considered.

Case (i). Suppose at least one number $i, 0 \leqslant i \leqslant 12$, and at least one number $j, 13 \leqslant j \leqslant 25$, are contained in the set $\left\{x_{0}, \ldots, x_{12}\right\}$. Then obviously $G_{1}=\left\langle s, \beta^{-1} s \beta\right\rangle$ fixes $L_{26}$ and is transitive on the remaining lines through the origin. Then $\left\langle G, G_{1}\right\rangle$ is doubly transitive on the lines of $\pi_{H}$ through the origin.

Case (ii). Suppose $\left\{x_{0}, \ldots, x_{12}\right\}$ consists of the numbers $\{13,14, \ldots, 25\}$. Then the action of the restriction of $r^{-1} \beta^{-1} s \beta r$ to the lines $L_{i}$ of $\pi_{H}$ through the origin is given by

$$
r^{-1} \beta^{-1} s \beta r:(26)(0)\left(z_{0}, z_{1}, \ldots, z_{12}\right)\left(a_{0}, a_{1}, \ldots, a_{12}\right)
$$

where $L_{z_{i}}=L_{13+j} r$ for some $j, 0 \leqslant j \leqslant 12$.

Since $L_{19} r=L_{2}, L_{17} r=L_{16}, L_{13} r=L_{27}$ we find that $\left\{z_{0}, z_{1}, \ldots, z_{12}\right\}$ contains 2,16 and 27 . This implies that $G_{2}=\left\langle s, r^{-1} \beta^{-1} s \beta r\right\rangle$ fixes $L_{26}$ and is transitive on the remaining lines of $\pi_{H}$ through the origin. Here again, the group $\left\langle G, G_{2}\right\rangle$ is doubly transitive on the lines of $\pi_{H}$ through the origin.

Similarly we may conclude that the collineation group of $\pi_{H}$ is doubly transitive on the lines of $\pi_{H}$ through the origin if $\beta$ fixes $L_{26}$ and maps $L_{27}$ onto $L_{13}$.

Since the order 27 of $\pi_{H}$ is odd and $27 \not \equiv 1(\bmod 8)$, the double transitivity implies that $\pi_{H}$ is Desarguesian [3, p. 217]. This is a contradiction since $\pi_{H}$ is non-Desarguesian. From this contradiction the truth of lemma follows.

Definition 5.2. If every collineation of $\pi_{H}$ fixing a line $L_{x}$ also fixes $L_{y}$, then $L_{y}$ is called a companion of $L_{x}$. 
LemMa 5.3. Every line $L_{x}$ of $\pi_{H}$ through the origin has a unique companion.

Proof. By Theorem 5.1 any collineation that fixes $L_{26}$ also fixes $L_{27}$ and $L_{27}$ is, therefore, a companion of $L_{26}$. Since $s$ fixes $L_{26}$ and $L_{27}$ and moves the remaining lines through the origin, none of the lines through the origin other than $L_{27}$ can be a companion of $L_{26}$. Thus $L_{27}$ is the unique companion of $L_{26}$. From the transitivity of $G$ on the lines $L_{i}$, we get that every line $L_{x}$ has a unique companion $L_{y}$. We may also remark that if $L_{y}$ is a companion of $L_{x}$ then $L_{x}$ is a companion of $L_{y}$.

LEMma 5.4. $\pi_{H}$ has a collineation the restriction of which to the lines $L_{i}$ fixes each line of any two pairs of companions and moves the remaining lines through the origin.

Proof. Follows from Lemma 5.3, structure of $h^{2}$ and $s$ and the transitivity of $G$ on the lines $L_{i}$.

LEMMA 5.5. If $\pi_{N}$ is Hering's plane, then the line $V_{k}$ is the unique companion of $V_{x}$ where $k \equiv x+14(\bmod 28)$.

Proof. Suppose $\pi_{N}$ is Hering's plane. Then there is a collineation $\gamma$ whose action restricted to the lines $V_{i}$ is given by

$$
\gamma:(x)(y)\left(a_{0}, a_{1}, \ldots, a_{12}\right)\left(b_{0}, b_{1}, \ldots, b_{12}\right)
$$

where $V_{y}$ is the unique companion of $V_{x}$. Then $T^{y-x} \gamma T^{x-y}$ fixes $V_{x}$ and $V_{j}$ where $j \equiv 2 x-y(\bmod 28)$, moving the remaining lines. In view of Lemma 5.3, $V_{j}$ must be $V_{y}$. This is possible only when $y \equiv 2 x-y(\bmod 28)$ which gives $2 x \equiv 2 y(\bmod 28)$, since $x \neq y$. This implies $y=x+14(\bmod 28)$.

THEOREM 5.6. $\pi_{N}$ is not isomorphic to $\pi_{H}$.

Proof. If $\pi_{N}$ is isomorphic to $\pi_{H}$, then in view of Lemmas 5.4 and 5.5 , $\pi_{N}$ has a collineation fixing $V_{0}, V_{14}, V_{1}$ and $V_{15}$ and moving the remaining lines through the origin. But this is not possible by Lemma 4.2 . Hence $\pi_{N}$ is not isomorphic to $\pi_{H}$.

\section{REFERENCES}

1. J. André, Uber nicht-Desarguessche Ebenen mit transitiver Translationsgruppe, Math. Z. 60 (1954), 156-186. MR 16, 64.

2. R. H. Bruck and R. C. Bose, The construction of translation planes from projective spaces, J. Algebra 1 (1964), 85-102. MR 28 \#4414.

3. P. Dembowski, Finite geometries, Ergebnisse der Mathematik und ihrer Grenzgebiete, Band 44, Springer-Verlag, Berlin and New York, 1968. MR 38 \# 1597.

4. D. A. Foulser, The flag-transitive collineation groups of the finite Desarguesian affine planes, Canad. J. Math. 16 (1964), 443-472. MR 29 \#3549.

5. - Solvable flag transitive affine groups, Math. Z. 86 (1964), 191-204. MR 30 \#1190.

6. M. Hall, Jr., The theory of groups, Macmillan, New York, 1959. MR 21 \#1996.

7. C. H. Hering, Eine nicht-desarguessche zweifach transitive affine Ebene der Ordnung 27, Abh. Math. Sem. Univ. Hamburg 34 (1969/70), 203-208. MR 42 \#3390.

8. D. R. Hughes, Review of some results in collineation groups, Proc. Sympos. Pure Math., vol. 1, Amer. Math. Soc., Providence, R.I., 1959, pp. 42-55. MR 22 \#7053.

9. T. G. Ostrom, Homologies in translation planes, Proc. London Math. Soc. (3) 26 (1973), 605-629. MR 48 \#2893. 
10. M. L. Narayana Rao, A flag transitive plane of order 49, Proc. Amer. Math. Soc. 32 (1972), 256-262. MR 44 \# 7428.

11. _ A class of flag transitive planes, Proc. Amer. Math. Soc. 39 (1973), 51-56. MR 47 \#4132.

12. G. Pickert, Projecktive Ebenen, Die Grundlehren der math. Wissenschaften, Band 80 , Springer-Verlag, Berlin, 1955. MR 17, 399.

13. A. Wagner, On finite affine line transitive planes, Math. Z. 87 (1965), 1-11. MR 30 \#2391.

Department of Mathematics, Osmania University, Hyderabad-500007, India 